\title{
The Potential of Citizen- Driven Monitoring of Freshwater Snails in Schistosomiasis Research
}

JEF BREES (10)

TINE HUYSE (D)

JULIUS TUMUSIIME (D)

GRACE KAGORO-RUGUNDA (D)

DAISY NAMIREMBE
FAITH MUGABI

VIOLA NYAKATO (D)

MAXSON KENNETH ANYOLITHO (D)

CASIM UMBA TOLO DD

LIESBET JACOBS (D)

*Author affiliations can be found in the back matter of this article

\section{ABSTRACT}

Schistosomiasis is a tropical parasitic disease affecting more than 200 million people worldwide, predominantly in Africa. The World Health Organization recently highlighted the importance of targeted control of the freshwater snails acting as intermediate hosts for the parasites causing schistosomiasis. However, because of a shortage of trained experts and resources, detailed information on spatiotemporal snail distributions, which is needed for targeted control measures, is often missing. We explore the potential of citizen science to build these much-needed datasets through fine-grained, frequent snail sampling. We trained a network of 25 citizen scientists to weekly report on snail host abundances in 77 predefined water contact sites in and around Lake Albert (western Uganda). Snail abundance, together with marked GPS locations, water chemistry parameters, and photographs of the identified snails are recorded and submitted using the freely available mobile phone application KoBoToolbox. Trained researchers then engage in remote, semi-automatic validation of the submissions, after which there is an opportunity to provide targeted feedback to the citizen scientists. Five months after the operationalisation of the network, a total of 570 reports were submitted and personalized feedback was given, resulting in lasting improvements in subsequent reporting and snail genus identification. The preliminary results show the possibility of citizen science to independently obtain reliable data on the presence of schistosome snail hosts. We therefore argue that citizen-driven monitoring on a high spatiotemporal resolution could help to generate the much-needed data to support local targeted snail control measures in remote and/or resource-limited environments. 


\section{INTRODUCTION}

Schistosomiasis is a neglected tropical disease caused by parasitic blood flukes of the genus Schistosoma. Ranking second only to malaria in terms of morbidity and prevalence, around 230 million people are affected worldwide (Colley et al. 2014), with the majority living in Sub-Saharan Africa (Gryseels et al. 2006). In Africa, the two main human schistosome species are Schistosoma haematobium and Schistosoma mansoni, which rely on freshwater snail species belonging to the genera Bulinus and Biomphalaria respectively. Chronic infections can lead to anaemia, haematuria, stunted growth, infertility, and fibrosis of the liver and urinary tract (Colley et al. 2014).

Since its introduction in the 1970s, mass drug administration (MDA) of the anthelmintic drug praziquantel has been central to schistosomiasis control in Sub-Saharan Africa (Gryseels et al. 2006). The Schistosomiasis Control Initiative was founded in 2002 with the aim of broadscale elimination of schistosomiasis by (mass) treatment of schoolchildren (Fenwick et al. 2009). However, MDA campaigns do not target the parasite's population in the very young or the elderly, nor those in the snail hosts. So despite these campaigns, reinfection after treatment enables the disease to re-emerge, resulting in a global prevalence as high as it was 50 years ago (Tchuem Tchuenté et al. 2017; Sokolow et al. 2018). Given this unsatisfactory outcome, the World Health Organization recently recommended complementing these MDA campaigns with targeted snail control (WHO 2017). Indeed, lowering snail densitiesthrough physical, chemical or biological measures-to a point where snail death rates exceed snail infection rates, would break the schistosome's life cycle (Sokolow et al. 2015, 2018). Efficiently targeting the snail host requires foremost a detailed understanding of its spatiotemporal dynamics (Shiff 2017; Tchuem Tchuenté et al. 2017). However, the collection of high-resolution distribution data is hampered by a scarcity in resources and malacological expertise (Shiff 2017; Tchuem Tchuenté et al., 2017; World Health Organization 2017).

Within (bio)geographic research, citizen science has been successfully implemented to address data collection bottlenecks. In Sub-Saharan Africa, earlier studies are available in the fields of hydrological monitoring (Gomani et al. 2010; Weeser et al. 2018) and natural hazards (Jacobs et al. 2019). In the field of vector-borne diseases, citizenbased platforms have contributed to unprecedented datasets on arthropod vectors that transmit diseases like malaria, Lyme disease, yellow fever, and Zika (Hamer et al. 2018; Nieto et al. 2018; Eritja et al. 2019). Despite these encouraging results, this approach to vector monitoring is still rare in Sub-Saharan Africa (Ashepet et al. 2021) and is to our knowledge not yet applied within the field of schistosomiasis research.

Here we present the setup of a citizen scientist network reporting on snail host abundances in a highly endemic area in western Uganda, where the prevalence of schistosomiasis can reach 90\% in the human population (Kabatereine et al. 2004). Apart from schistosomiasis, fasciolosis is endemic in Uganda. This disease is caused by liver flukes (Fasciola spp.) that are transmitted by freshwater snails of the genus Radix (Malatji et al. 2020). It predominantly affects sheep and cattle, but humans can also become infected. Given its economic impact on livestock industry in Uganda (Joan et al. 2015), we also include these snail species in our monitoring program.

Our pilot study explores key aspects in the development of a citizen science framework to monitor snail hosts for schistosomiasis and fasciolosis. The approach is strongly steered by an underlying research objective, namely understanding the spatial and temporal dynamics of snail host populations in a highly endemic, resource-poor region. The resulting requirements in data quality necessitate a structured systematic monitoring of snails over time in a diversity of habitat types. As such, our study differs from existing vector monitoring approaches, which are either opportunistic in nature (e.g., the incidental reporting of ticks in Nieto et al. 2018) or require less elaborate sampling procedures (compared with, for example, passive mosquito trapping in Braz Sousa et al. 2020).

Therefore, within this pilot study, special attention was paid to 1) selecting and training of the citizen scientists, 2) designing a data collection and validation protocol that minimises interpretation errors and facilitates data quality control, and 3) providing personalized feedback to the volunteers and assessing the feedback's effect on data quality.

\section{METHODOLOGY \\ WATER SITE SELECTION}

This study is situated around the southern part of Lake Albert and its surrounding upland catchments ( 750 $\mathrm{km}^{2}$ ) (Figure 1). The area is characterized by a diverse hydrological setting, covering small upland streams, larger rivers, swamps, stationary pools, artificial spring wells, lacustrine shorelines, and sheltered bays.

During a field exploration in February 2020, 77 water contact sites were selected for the monitoring program, covering topographic and hydrological gradients within the study area (Figure 1). A site was defined as a delineated section of a water body that is used for human activities, such as fishing or bathing, for at least some part of the year. For rivers and streams, a site was specified as a 


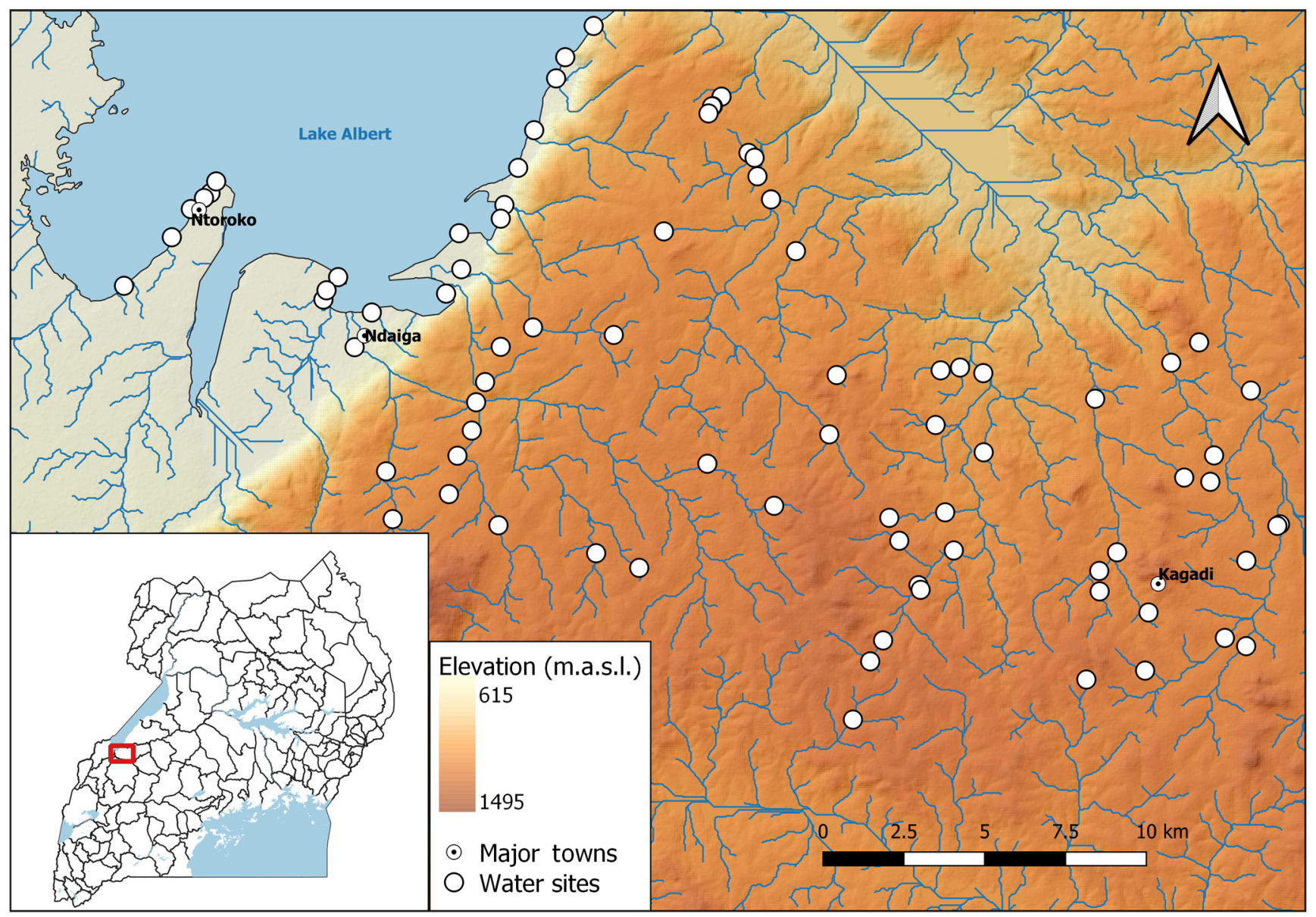

Figure 1 Overview map of the study area showing the selected water contact sites around the southern part of Lake Albert and upstream catchments. (Elevation data: Shuttle Radar Topography Mission (SRTM) downloaded from NASA's Earthdata Search, river network was extracted from the Digital Elevation Model).

10-meter section including both banks. Pools and ponds smaller than ca $100 \mathrm{~m}^{2}$ were considered in their entirety, while sites at larger water bodies and lakes were marked by a 10-meter section along the shoreline. Care was taken to avoid potential biogeochemical barriers (e.g., culverts, artificial substrate transitions, major hydrological steps) within a single water contact site. Their presence up- or downstream of the site was systematically recorded.

\section{DATA COLLECTION}

\section{Snail sampling and ancillary data collection}

Timed searches using a fine-meshed scoop net have been the standard procedure for snail host sampling over the past few decades (Sokolow et al. 2018; Perez-Saez et al. 2019). Actively searching through potential microhabitats results in an estimated abundance expressed as the number of snails caught per person per time unit. Although highly valuable to capture seasonal variability within a single population, some possible concerns arise when comparing absolute abundances between different sites owing to the varying sampling efficiencies of individuals and different habitat typologies (Sokolow et al. 2018; Perez-Saez et al. 2019). Random quadrat sampling involves replicate, exhaustive sampling of a small, delineated surface area to estimate a population's absolute density. Despite enabling a direct comparison of the obtained abundances across different sites and individuals, this strategy is far more labour-intensive (Perez-Saez et al. 2019).

Given the widespread use and overall practical advantage of timed searches over the more cumbersome quadrat sampling, we opted for the former strategy. Every citizen scientist is linked to two to four water sites in which they sample the populations of the three main snail genera acting as intermediate hosts for parasitic flatworms in the area, namely Biomphalaria and Bulinus for Schistosoma spp., and Radix or related lymnaeid species for Fasciola spp. transmission. Sampling is done on a weekly basis for an initial period of three years. Additionally, the citizen scientists report on several chemical parameters of the water, including $\mathrm{pH}$, nitrate $\left(\mathrm{NO}_{3}\right)$ and chlorine $\left(\mathrm{Cl}_{2}\right)$ 
concentrations, carbonate hardness $(\mathrm{KH})$, general hardness $(\mathrm{GH})$ and water temperature, as well as the human activities going on at the time of sampling (e.g., fishing, bathing).

\section{Equipment and data collection protocol}

The data was collected using Huawei Y7 smartphones. These devices were chosen for their relatively long battery life (3500 mAh), 13-megapixel rear camera, and GPS functionality at a reasonably low price. The data reporting was done through the freely accessible KoBoToolbox platform, which is specifically designed for data collection in remote environments (KoBoToolbox 2014). Questionnaires can be developed on a cloud-based interface, and the mobile application allows participants to fill out the questionnaires offline and submit them to the server whenever they have an internet connection.

All citizen scientists (see below for selection) received the necessary measurement equipment (Figure 2a) and protective gear to safely perform the monitoring. The equipment is listed together with its respective purpose in Table 1.

The citizen scientists are asked to sample all water contact sites under their responsibility once a week. A single questionnaire with a fixed chronological sequence was developed to standardize this sampling across all sites and citizen scientists (https://github.com/ATRAP-UG/KOBO_ Questionnaire). Multiple-choice questions are favoured over open questions, and when possible, open input is restricted

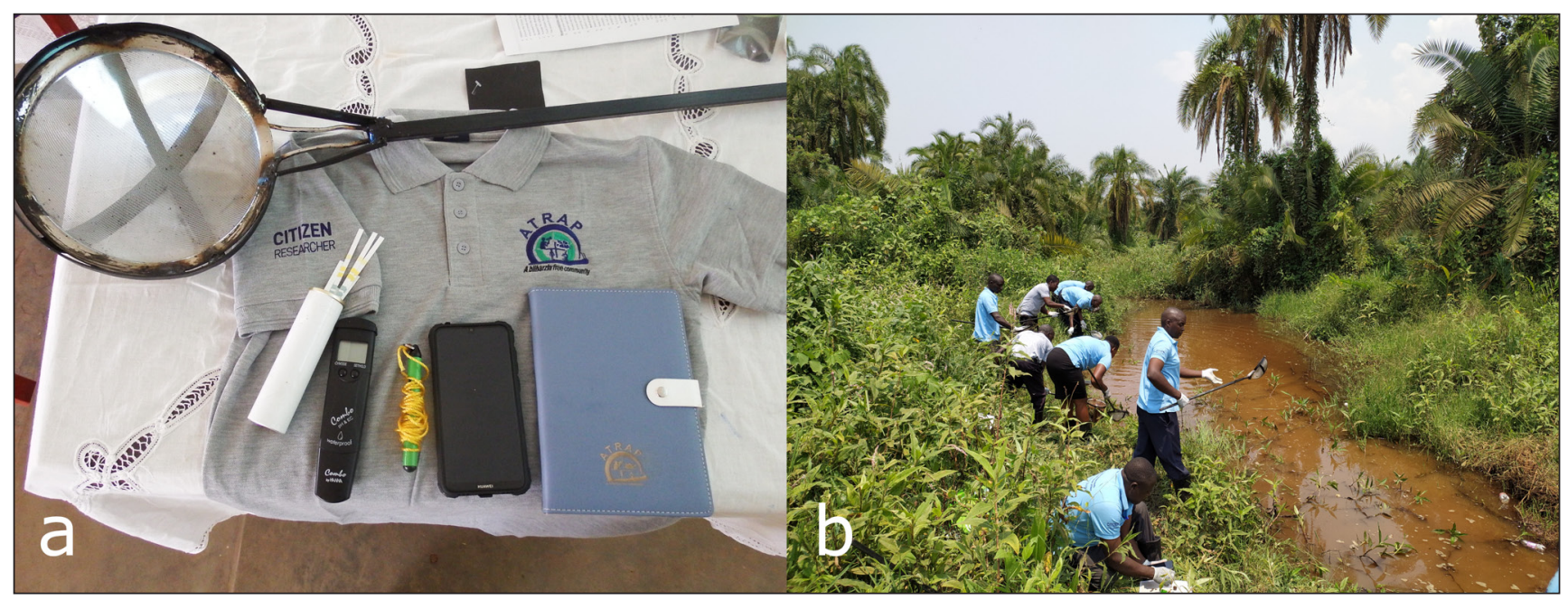

Figure $2(\boldsymbol{a})$ Overview of the equipment provided to the citizen scientists: fine-meshed scoop net, ATRAP project t-shirt, water chemistry test strips, stream thermometer, smartphone, diary and Hanna Instruments pocket tester. Latex gloves, rubber boots and the laminated sheet of millimetre paper are not shown in the photograph. (b) In-field sampling exercise during the two-day training course for the citizen scientists in Kagadi.

\begin{tabular}{lll}
\hline EQUIPMENT & PURPOSE & DETAILS \\
\hline Protective gloves & $\begin{array}{l}\text { Protection: avoid contact with potentially infected } \\
\text { water }\end{array}$ & $\begin{array}{l}\text { Disposable latex gloves + heavy-duty long } \\
\text { sleeve rubber gloves }\end{array}$ \\
\hline Gumboots & $\begin{array}{l}\text { Protection: avoid contact with potentially infected } \\
\text { water }\end{array}$ & $/$ \\
\hline Stream thermometer & Measurement temperature & Accuracy of $1^{\circ} \mathrm{C}$ \\
\hline Single-use test strips & $\begin{array}{l}\text { Measurement } \mathrm{pH}, \text { nitrate }\left(\mathrm{NO}_{3}\right) \text { and chlorine }(\mathrm{Cl}) \\
\text { concentrations, carbonate hardness }(\mathrm{KH}) \text {, general } \\
\text { hardness }(\mathrm{GH})\end{array}$ & $/$ \\
\hline Hanna Instruments HI98129 pocket tester & $\begin{array}{l}\text { Measurement of water temperature, } \mathrm{pH}, \text { electrical } \\
\text { conductivity (EC), and total dissolved solids (TDS) }\end{array}$ & $\begin{array}{l}\text { Five citizen scientists were equipped with } \\
\text { this device, at different locations and } \\
\text { hydrological settings to encompass the } \\
\text { diversity within the study area. }\end{array}$ \\
\hline
\end{tabular}

Table 1 Overview of the sampling equipment of the citizen scientists. In addition to the equipment mentioned here, every citizen scientist received a personal notebook to record any information they considered relevant. 
to predefined ranges to minimise erroneous entries. The use of skip logic allows only those questions applicable for the particular observations to be shown.

At a water site, the citizen scientists start the questionnaire by identifying themselves and the site, and confirming that they are wearing the protective equipment. The date and time are specified, after which the citizen scientist is prompted to take an overview picture of the water site and its immediate surroundings. Next, a GPS point of the citizen scientist's location at the site is marked, followed by a series of questions about the human activities taking place at the site and the number of people involved. Subsequently, the stream thermometer is placed in the water while a test strip is dipped in the water for one second and allowed to adjust to the water chemistry for one minute. The citizen scientists are asked to place the strip alongside the corresponding colour-chart and take a photograph, eliminating the need for interpretation in the field. Hereafter, the thermometer is taken out of the water and the read-off is entered directly in the questionnaire. At this point, the citizen scientists with a Hanna Instruments pocket tester are prompted to report the measurements for temperature, $\mathrm{pH}$, electrical conductivity (EC), and total dissolved solids (TDS). After this, another question for the current time marks the start of the snail sampling, which is a 30-minute scooping effort. All sampled snails are kept aside, and the number of scoops is counted. Afterwards, the current time is again recorded, and the citizen scientist is asked to enter the number of scoops. A snail-determination guide helps the citizen scientist to distinguish Biomphalaria, Bulinus, and Radix from all other freshwater snail genera (hereafter called the Pool group). The specimens belonging to each genus are counted and placed on a laminated sheet of millimetre paper for scale, after which photographs are taken and uploaded to the questionnaire. Finally, the completed questionnaire is saved and can be submitted whenever a stable internet connection is found. Once uploaded, the submitted questionnaire is referred to as a report.

\section{Report validation}

To ensure standardized data quality control across the large number of reports submitted over time, a validation protocol was developed in Python (https://github.com/ATRAPUG/Report-Validation). One part of the validation is performed automatically, using solely the (meta-)data in each report, whereas the second part requires input from a researcher. This initial validation does not guarantee flawless reports, but rather flags evident mistakes.

The automatic part of the validation starts with an ID check to verify whether the assigned smartphone was used to report on a site. This ensures that the citizen scientists report only on their own sites. Second, the GPS location marked in the report is compared with the true location mapped during the field exploration campaign. The report's location is flagged whenever the latitudinal and/or longitudinal distance to the true location exceeds 200 meters. This threshold was chosen to account for potential shifts of the site owing to varying water levels throughout the year, while remaining a useful indicator for potentially wrongly sampled locations. Third, the timestamp questions directly before and after the scooping section allow the verification of the true sampling time. As the citizen scientists are asked to scoop for 30 minutes, any scooping effort shorter than 20 minutes or longer than 40 minutes is flagged. Reports indicating temperatures outside of the expected range of $10-35^{\circ} \mathrm{C}$ (Kazibwe et al. 2006; Rowel et al. 2015) are also flagged.

The second part of the validation requires input from an experienced researcher. First, the researcher is shown the photograph of the water test strip and asked to enter the values for $\mathrm{NO}_{3}, \mathrm{pH}, \mathrm{GH}, \mathrm{KH}$, and $\mathrm{Cl}_{2}$. Whenever the photograph does not allow an accurate read-off, it is flagged as invalid. Secondly, regarding verification of the reported snails, the validation protocol focusses on verifying the genus classification. The submitted photographs of the classified snail groups (Biomphalaria, Bulinus, Radix, and Pool) are displayed and visually checked. In case of a misclassification of one of the specimens, the concerned snail group is flagged, resulting in a false positive report for that respective snail group. The Pool group is considered invalid when it contains specimens that should have been in one of the three snail host groups. When this occurs, the researcher specifies which of the three snail genera of interest is wrongly reported under the Pool group, resulting in a false negative report for that respective snail group.

\section{SELECTION AND TRAINING OF THE CITIZEN SCIENTISTS}

The citizen scientist network was set up under the project Action Towards Reducing Aquatic Snail Borne Parasitic Diseases (ATRAP) (https://www.citizenscienceuganda.info/ atrap-project-description.html) through a collaborative effort between the Mbarara University of Science and Technology (MUST) in Uganda, and the Royal Museum for Central Africa (RMCA) and KU Leuven in Belgium. In February 2020, the participants were selected based on a list of criteria that were formulated by the entire project team, which consisted of social scientists, biologists, epidemiologists, and geographers. These criteria include administrative requirements (e.g., minimum 18 years old) and less stringent favourable qualifications, such as gender balance, area of residence, motivation, and volunteer experience in the community (see Supplemental File 1). 
The first stage of selection was to organize meetings with leaders from the administrative and political levels, and with health officers and representatives of religious or cultural groups. These leaders know their communities thoroughly and are therefore well placed to indicate who might be interested, motivated, able to participate, and trusted by the community. By devoting ample time for mutual exchange, the views of the community leaders on the project could be probed and integrated in the subsequent steps of setting up the network. The community leaders were asked to jointly nominate and mobilize potential volunteers from within their sub-county based on the predefined set of criteria. Through face-toface interviews conducted by the research team, a final group of 25 citizen scientists was formed. The citizen scientists were predominantly male (68\%), between 23 and 49 years of age, with an average age of 37 . The most common occupations were farming, fishing, and shop keeping. A vast majority (84\%) had already been involved in voluntary work through, e.g., participation in the Village Health Teams (VHT) and Red Cross projects.

Next, the 25 citizen scientists were invited to a twoday training course in Kagadi. The schedule and content for this training was jointly designed by the entire ATRAP team. The first day consisted of an in-depth explanation of schistosomiasis and the relevance of the citizen scientist's involvement. Furthermore, an introduction on how to use the smartphone was given. Finally, the use of the thermometer, water test strips and pocket tester were demonstrated, and an entire protocol was jointly run through with mock data. The second day started with a training on snail identification. Snail specimens preserved in ethanol were used to illustrate some characteristic traits of the snail hosts of interest, and to teach participants how to distinguish the specimens of interest from other freshwater snail genera. This was followed by two in-field monitoring sessions to put everything into practice (Figure $2 b$ ), and feedback was given on the spot based on incoming reports. During the two weeks following the two-day training course, each citizen scientist was accompanied to their water contact sites by a snail expert, and at least one full questionnaire was jointly completed. This way, additional feedback could be tailored to the experience and provided on the spot.

Citizen scientists are compensated 75,000 UGX (ca. 17 euros) on a monthly basis for transportation costs and mobile data to submit the reports. This sum is thus intended as compensation rather than as a salary and was not specified to the candidates during the selection process. This monthly reimbursement is of a similar magnitude as that of another citizen scientist network reporting on natural hazards in the Rwenzori region (Jacobs et al. 2019).
The citizen scientist network became fully operational on 04 March 2020. In the initial three weeks between operationalisation and a temporary suspension due to the Covid-19 pandemic on 28 March 2020, a total of 224 reports were submitted. These initial reports were validated as described earlier, and based on the results, tailored feedback was provided to the citizen scientists in person. The network restarted the monitoring activities on 24 June 2020 , leading to an additional 346 reports submitted by 05 August 2020.

\section{RESULTS}

Over the course of nine active sampling weeks between March and August 2020, a total of 570 reports were submitted. Given the 77 water contact sites, we would ideally expect the total number of reports to be 693 . The missing reports result from a variety of different causes, such as temporary inaccessibility of the sites, or personal inability to sample due to illness or other pressing matters. In this section, we report on the most common errors, the usefulness of the validation protocol, the impact of personalized feedback, and the prospects of the collected data for further research.

\section{REPORT VALIDATION: MOST COMMON ERRORS AND THE EFFECT OF FEEDBACK}

On average, the citizen scientists spent 42 minutes sampling a single site. An overview of the validation results is given in Table 2. Apart from the location error, all errors showed a decrease after the personalized feedback provided by 24 June 2020. It was found that incorrect location settings on the smartphones of four citizen scientists led to highly inaccurate GPS readings in 30 of the 346 reports submitted after the suspension, thereby artificially raising the locations flagged as invalid. Hence, these reports were not considered in the following analysis. The issue has since been resolved.

Regarding the snail identifications, for all genera, false positive reports were more common than false negatives (Table 2). In other words, there were more reports in which snails were misclassified as Biomphalaria, Bulinus or Radix than reports in which snails belonging to these respective genera were found in the Pool class. The Bulinus identification resulted in the most mistakes, with up to $80 \%$ false positive reports being identified after the initial start-up.

To explore the effect of feedback on the level of individual citizen scientists, the average number of errors per ten submissions for each of the 25 volunteers, both before and after the feedback, is shown (Figure 3). First, it should be noted that in each report, a maximum of eight 


\begin{tabular}{|c|c|c|c|c|c|c|}
\hline ERROR & $\begin{array}{l}\text { INVALID REPORTS } \\
\text { (\%) PRIOR }\end{array}$ & $\begin{array}{l}\text { INVALID } \\
\text { REPORTS } \\
\text { (\%) AFTER }\end{array}$ & $\begin{array}{l}\text { FALSE POSITIVE } \\
\text { (\%) PRIOR }\end{array}$ & $\begin{array}{l}\text { FALSE POSITIVE } \\
\text { (\%) AFTER }\end{array}$ & $\begin{array}{l}\text { FALSE NEGATIVE } \\
\text { (\%) PRIOR }\end{array}$ & $\begin{array}{l}\text { FALSE NEGATIVE } \\
\text { (\%) AFTER }\end{array}$ \\
\hline ID & 0 & 0 & N.A. & & & \\
\hline Location & 4 & 13 & & & & \\
\hline Scoop time & 7 & 5 & & & & \\
\hline Test strip & 8 & 4 & & & & \\
\hline Biomphalaria & 4 & 2 & 5 & 2 & 4 & 1 \\
\hline Bulinus & 11 & 3 & 80 & 32 & 0 & 0 \\
\hline Radix & 6 & 4 & 17 & 11 & 0 & 0 \\
\hline
\end{tabular}

Table 2 Overview of the validation results showing the percentage of reports containing a specific error, both before and after the personalized feedback. For the identification of the three snail genera of interest, the percentages of false positive and false negative reports are also provided. $(n=570)$.

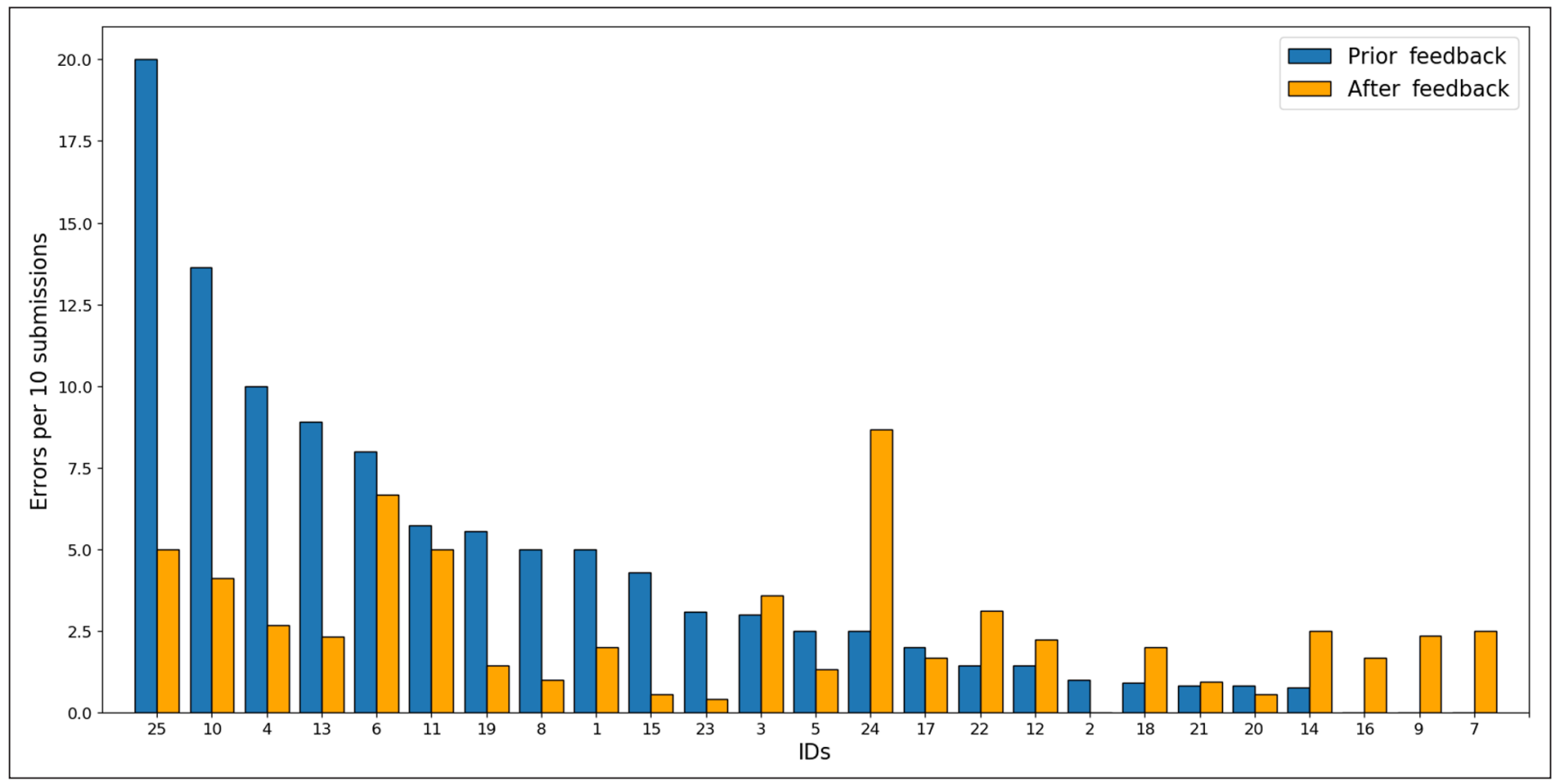

Figure 3 The average number of errors per 10 reports for each of the 25 citizen scientists, both before and after the personalized feedback. Note that in each report, a maximum of eight potential errors can be flagged.

potential errors can be identified. Prior to the feedback, most of the citizen scientists (64\%) made on average fewer than five errors per ten submissions. Another $28 \%$ made between five and ten errors per ten submissions, with a final $8 \%$ making on average more than ten errors per ten submissions. These results improved considerably after the feedback was given, with the most notable changes amongst the citizen scientists with initially the highest error rates. The fraction of citizen scientists making on average less than five errors per ten submissions increased to $92 \%$, with only $8 \%$ averaging between five and ten errors. For all snail genera, classifications improved after feedback. Bulinus remained the most difficult genus to identify, but the number of false positive reports reduced considerably to $32 \%$ after feedback. Contrastingly, Biomphalaria classifications were remarkably accurate, both in terms of false positive (2\%) as well as false negative reports (1\%). The citizen scientist with ID 24 misinterpreted the feedback on snail identification and therefore made more errors on this aspect than before. This issue has since been addressed and resolved.

\section{EXEMPLIFYING RESULTS: SAMPLED SNAIL HOST ABUNDANCES}

To further explore the potential of this approach, some exemplifying results from the citizen-driven dataset are 
shown, such as a map of the average sampled snail host abundances in March 2020 (Figure 4). At that time, sites along the shores of Lake Albert were reported to have far lower snail abundances than inland river sites, or even no snails at all. Meanwhile, some inland river sites had total snail abundances of more than 150 specimens per 30 minutes of scooping. Most of the water contact sites had populations dominated by Biomphalaria sp., followed by Radix sp., with only a few sites reporting Bulinus sp.

In addition to assessing spatial distributions at a certain time, the weekly sampling allows us to monitor population dynamics at a high temporal resolution. For example, Figure 5 demonstrates variations in abundances of Biomphalaria sp. at four sites sampled by a single citizen scientist, with some sites showing stable abundances and others characterized by larger fluctuations.

\section{DISCUSSION}

We explored the potential of citizen science as a proof of concept to upscale snail sampling and address the pressing need for spatiotemporally dense information on freshwater snail host abundances. In the following sections, we discuss some key findings from our pilot study and frame them in the wider context of citizen science in remote and/or resource-limited environments.

\section{DATA QUALITY AND RESEARCH POTENTIAL OF THE PILOT STUDY}

Concerns surrounding citizen science datasets often revolve around the possibility of inaccurate or incomplete measurements, as well as the lack of validation to control for potential biases (Flanagin and Metzger 2008; Pocock et al. 2019). In our research design, we distinguished two levels of abstraction between the actual in situ situation in the field and the information received from the citizen scientists. The first can be defined as an observation bias, which is the difference between the actual in situ conditions and the sampled observation made by the citizen scientists, e.g., due to incomplete observations. The second can be termed a reporting bias-the difference between that sampled observation and the information reported through the questionnaire, e.g., due to errors

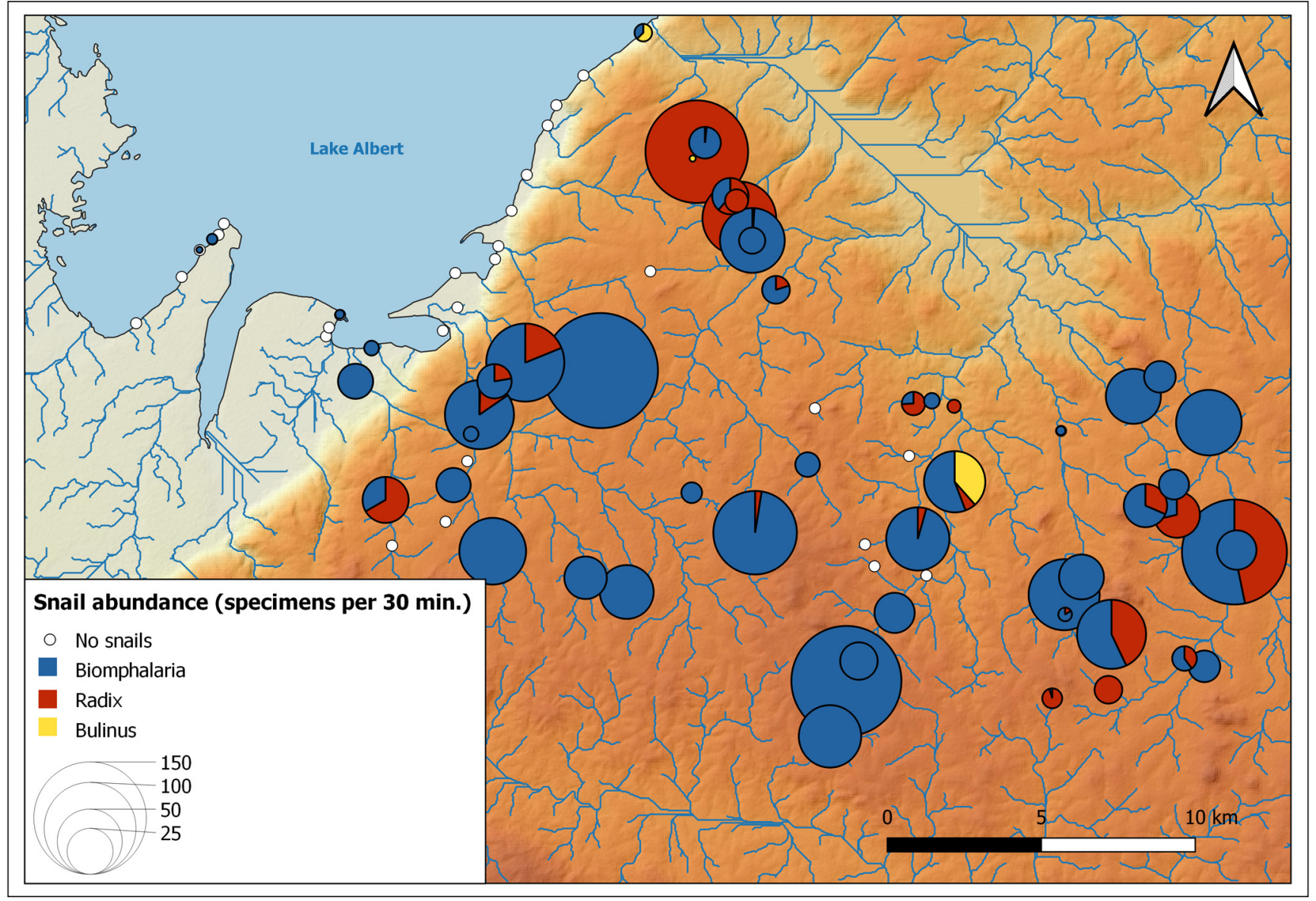

Figure 4 Map of the average reported abundances of snail species belonging to Biomphalaria, Radix, and Bulinus in March 2020. White circles mark sites where no species belonging to any of the three genera of interest were found. 


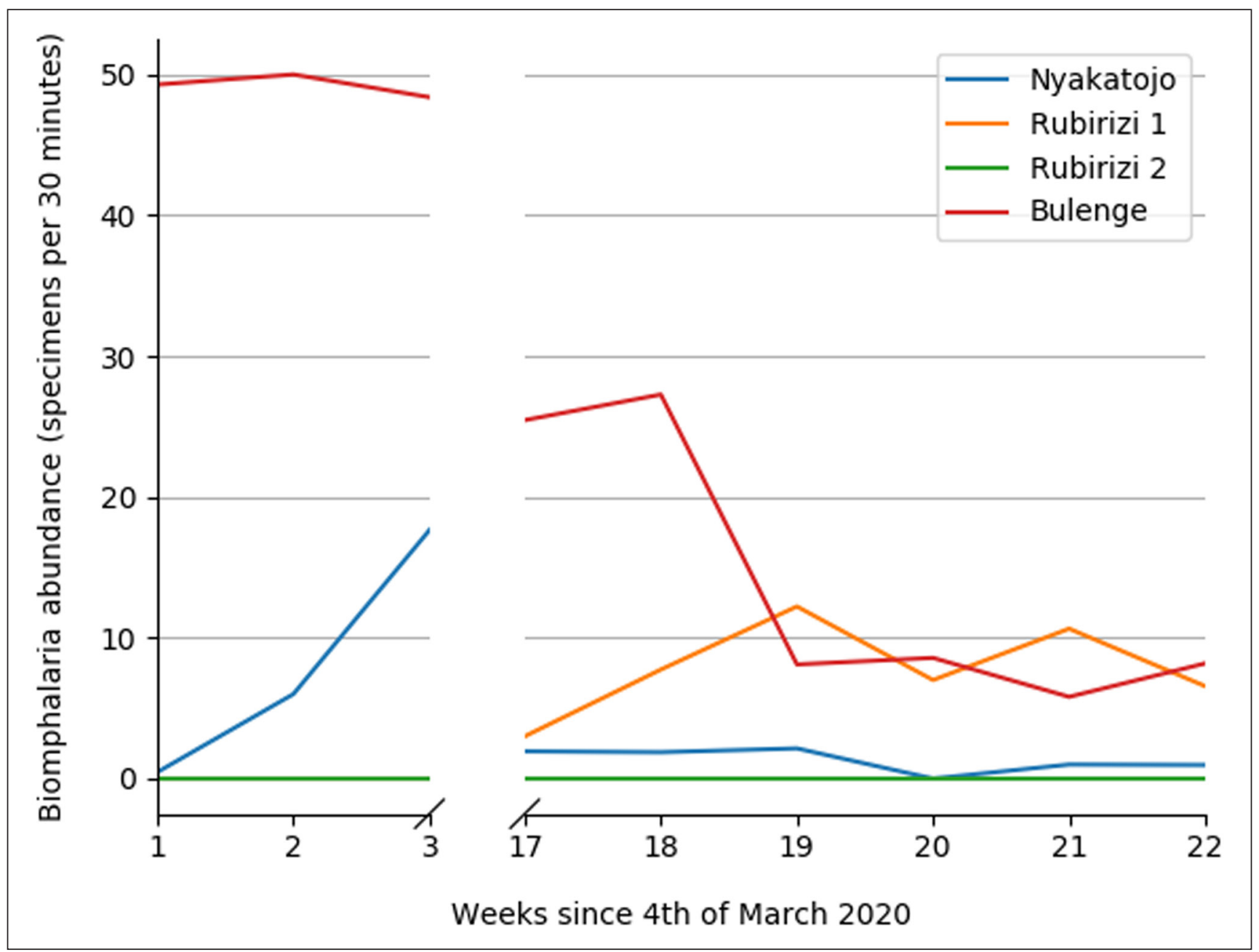

Figure 5 Sampled Biomphalaria sp. abundances through time at four sites monitored by a single citizen scientist (ID 23).

in the interpretation of the observations or to mistakes when filling in the questionnaire. Ideally, both biases are minimized, or at least accounted for, when interpreting the reported information as a sample of the real-world situation.

Reporting biases were minimized by the layout of the Kobo questionnaire and the validation protocol, for example by restricting entries to realistic ranges. Furthermore, the design of the protocol and the semi-automatic validation allowed us to provide tailored, individual feedback to the citizen scientists where necessary. Similar to other case studies (e.g., van der Wal et al. 2016), the results of the feedback showed clear improvements in the average reporting quality, with the most notable changes among the initially least-performing volunteers (Figure 3). Despite these advancements, the identification of Bulinus sp. remained rather poor with $32 \%$ false positive reports. This is likely the result of the higher plasticity in morphology compared with the other genera of interest (Brown 1994). Conversely, the identification of Biomphalaria species was by far the best, with only $2 \%$ false positive reports and
1\% false negative reports (Table 2). This is very promising because intestinal schistosomiasis, which is transmitted by Biomphalaria snails, is highly endemic in the study area, whereas urinary schistosomiasis, transmitted by Bulinus species, is considered of minimal public health significance (Kabatereine et al. 2007). The moderately positive results for Radix open the possibility for future fasciolosis research.

Assessing-and subsequently minimizing-the observation bias is less straightforward because independent validation on the ground is necessary. Similar to other studies contrasting citizen-driven with professionally collected datasets (e.g., Krabbenhoft and Kashian 2020), we intend to compare the citizen science dataset with a monthly sampling campaign at the same water contact sites by an experienced malacologist. This comparison will provide more conclusive evidence whether citizendriven data can accurately reflect the spatiotemporal population dynamics, i.e., abundances of snail hosts, and test the reliability of the water chemistry measurements. That way, we will be able to attribute spatiotemporal trends in sampled abundances to either actual variations 
in the snails' dynamics or to differences in the citizen scientists' observational biases. For example, contrary to our expectations, the abundance of Biomphalaria sp. in March 2020 was very high in upland areas where schistosomiasis is generally absent, but very low at the lakeside (Figure 4), where the prevalence of schistosomiasis is highest (Kabatereine et al. 2004). Correctly interpreting and attributing these differences in abundance (e.g., to seasonal or spatial drivers) requires assessing any effects of observational bias on the reported abundances.

Given the large improvements after feedback and the highly accurate identification of Biomphalaria snails, we can cautiously conclude that the current citizen scientist network can generate relevant information on putative transmission sites of intestinal schistosomiasis through a continuously growing dataset, unprecedented in terms of spatiotemporal resolution. The next step will be to quantify the observation biases, necessary to assess the completeness of the observations and to validate the abundance data. If successful, the dataset can form the basis for research on snail population dynamics and species distribution modelling.

\section{BEYOND THE PILOT STUDY: BROADER PERSPECTIVES FOR CITIZEN SCIENCE AND VECTOR MONITORING IN RESOURCE-LIMITED SETTINGS}

Similar to earlier studies on the potential of citizen science in monitoring vectors for human pathogens (e.g., Porter et al. 2019; Braz Sousa et al. 2020), this pilot study was incentivized by the observation that the labour- and resource-intensive nature of vector monitoring by experts hampers multiyear large-scale observations and the setup of effective control programs. An additional lack of (inter) national standards further complicates the monitoring of freshwater snail hosts (Stothard et al. 2017; PerezSaez et al. 2019; Maes et al. 2021). Given the need for spatiotemporally dense observations, we designed a systematic sampling and classification strategy that can be executed by a selected group of trained volunteers.

Although still in its exploratory stages, the proposed citizen science approach could be a sound candidate for the further development of a standardized snail host surveillance design at larger scales for several reasons. First, the relatively low amount of resources required for the setup and functioning of the citizen scientist network makes the approach applicable to a variety of settings and sustainable for multiyear surveying. Second, the approach can be implemented across different regions provided that the same cloud-based surveying protocol and a consistent training layout is adopted. This implies that datasets from different networks operating across various regions could be identically formatted and stored centrally for easy access and analysis.

Setting up a citizen scientist network should, however, not be regarded as a quick fix. Ample consideration should be given to the research design to maximize the quality of the resulting dataset. Generally, one should aim to minimize the likelihood of misinterpretation during the data collection/reporting by anticipating and accounting for possible mistakes in the sampling protocol, and designing an in-depth training for the citizen scientists. Additionally, the implementation of a standardized validation protocol allows early identification of recurring mistakes and provides the necessary information for targeted feedback to the citizen scientists.

A citizen-driven approach also requires considerations that span beyond data-collection design and implementation. First, participant motivation is key to ensuring sustainable, long-term participant engagement (Pocock et al. 2019; Ashepet et al. 2021; West et al. 2021). Second, Pocock et al. (2019) identified the reluctance of uptake of citizen science by policy makers as an important institutional barrier for their implementation in East Africa. Aside from these two challenges, a citizen science approach does offer the added possibility for the volunteers to act as communicators between the professional researchers and the wider community audience (Jacobs et al. 2019; Pocock et al. 2019; Ashepet et al. 2021). Hence, ongoing research within the ATRAP project will also focus on these three aspects to pursue scientifically, societally, and policyrelevant citizen science.

\section{CONCLUSION}

In this study, we use a citizen-driven approach for snail sampling and abundance monitoring in the Lake Albert region, western Uganda. Methodological choices include a structured data-collection protocol, a well-considered citizen scientist recruitment and training strategy, as well as a validation procedure that minimizes reporting bias and allows for targeted feedback to the citizen scientists. Initial results show the potential of this approach to systematically perform complex tasks, including the identification of relevant snail genera and collection of ancillary data at an unprecedented spatial and temporal resolution in this resource-limited environment. Future steps will include efforts to quantify and minimize the observational bias and use the continuously expanding and validated dataset as the basis for research on snail host population dynamics. 


\section{DATA ACCESSIBILITY STATEMENT}

The structured questionnaire and validation script can be found on the @ATRAP-UG GitHub page. The validated submissions and their attached photographs are available under the CC BY 4.0 licence:

https://figshare.com/articles/dataset/The_potential_of_citizendriven_monitoring_of_freshwater_snails_as_hosts_for schistosomiasis_in_remote_environments/14755146

\section{SUPPLEMENTARY FILE}

The supplementary file for this article can be found as follows:

- Supplemental File 1. Minimum requirements and favourable qualifications for the selection of the citizen scientists. DOI: https://doi.org/10.5334/cstp.388.s1

\section{ETHICS AND CONSENT}

The Uganda National Council for Science and Technology (UNCST) has reviewed and approved the research activities in this project (reference number: NS128ES). Any personal information shared with the research team is treated as confidential, and when publishing data, anonymity is guaranteed.

\section{ACKNOWLEDGEMENTS}

This research is financed by the ATRAP project of the Development Cooperation program of the Royal Museum for Central Africa with support of the Directorate-General Development Cooperation and Humanitarian Aid. We thank Anton Van Rompaey for his support within the project. We also explicitly thank all citizen scientists for their invaluable contributions: Alinda Hassan, Chotum Friday, Atanasi Marisel, Opio Isingoma, Ategeka Augustine, Unimu Hadija, Fuwarwinyo Richard, Sebakara Fobious, Nyamahunge Imelda, Busnge Zabron, Tumusiime Janet, Nsenga David, Night Marygoret, Kyaligonza Noeline, Mwesige Robert, Barisigara Gard, Tusingwire Henry, Natabi Specioza, Nakingi Rose, Ategeka Rogers, Kamukama Josias, Nuwagaba Emmanuel, Tweheyo Julius, and Bahungirehe Cruzestom. This research would not have been possible without their enthusiasm.

\section{COMPETING INTERESTS}

The authors have no competing interests to declare.

\section{AUTHOR CONTRIBUTIONS}

$J B, T H, L J, C T, J T, V N, M A$, and GK conceived the ideas and designed the study; JB, JT, DN, FM, MA, and $L J$ conducted the fieldwork and collected the data with additional collaborators; JB analysed the data; and JB led the writing with assistance from all co-authors.

\section{AUTHOR AFFILIATIONS}

Jef Brees (D) orcid.org/0000-0001-7713-2632 Dept. of Biology, Royal Museum for Central Africa, BE; Dept. of Earth \& Environmental Sciences, KU Leuven, BE

Tine Huyse (D) orcid.org/0000-0001-8164-9757 Dept. of Biology, Royal Museum for Central Africa, BE

Julius Tumusiime (D) orcid.org/0000-0002-4822-1078 Dept. of Biology, Mbarara University of Science and Technology, UG

Grace Kagoro-Rugunda (D) orcid.org/0000-0002-4498-9135 Dept. of Biology, Mbarara University of Science and Technology, UG

Daisy Namirembe

Dept. of Biology, Mbarara University of Science and Technology, UG Faith Mugabi

Dept. of Human Development and Relational Sciences, Mbarara University of Science and Technology, UG

Viola Nyakato (D) orcid.org/0000-0003-1148-9959

Dept. of Human Development and Relational Sciences, Mbarara University of Science and Technology, UG

Maxson Kenneth Anyolitho (D) orcid.org/0000-0003-1421-1178 Dept. of Human Development and Relational Sciences, Mbarara University of Science and Technology, UG

Casim Umba Tolo (D) orcid.org/0000-0003-1807-3438 Dept. of Biology, Mbarara University of Science and Technology, UG

Liesbet Jacobs (iD orcid.org/0000-0002-1137-5372

Dept. of Earth \& Environmental Sciences, KU Leuven, BE; Institute for Biodiversity and Ecosystem Dynamics, University of Amsterdam, NL

\section{REFERENCES}

\section{Ashepet, MG, Jacobs, L, Van Oudheusden, M and Huyse, T.}

2021. Wicked solution for wicked problems: citizen science for vector-borne disease control in Africa. Trends in Parasitology, S1471492220302890. DOI: https://doi.org/10.1016/j. pt.2020.10.004

Braz Sousa, L, Fricker, SR, Doherty, SS, Webb, CE, Baldock, KL and Williams, CR. 2020. Citizen science and smartphone e-entomology enables low-cost upscaling of mosquito surveillance. Science of The Total Environment, 704: 135349. DOI: https://doi.org/10.1016/j.scitotenv.2019.135349

Brown, D. 1994. Freshwater Snails Of Africa And Their Medical Importance. $1^{\text {st }}$ ed. London, UK: Taylor \& Francis. DOI: https:// doi.org/10.1201/9781482295184 
Colley, DG, Bustinduy, AL, Secor, WE and King, CH. 2014. Human schistosomiasis. The Lancet, 383: 2253-2264. DOI: https://doi. org/10.1016/S0140-6736(13)61949-2

Eritja, R, Ruiz-Arrondo, I, Delacour-Estrella, S, Schaffner, F, Álvarez-Chachero, J, Bengoa, M, Puig, M-Á, Melero-Alcíbar, R, Oltra, A and Bartumeus, F. 2019. First detection of Aedes japonicus in Spain: an unexpected finding triggered by citizen science. Parasites \& Vectors, 12: 53. DOI: https://doi. org/10.1186/s13071-019-3317-y

Fenwick, A, Webster, JP, Bosque-Oliva, E, Blair, L, Fleming, FM, Zhang, Y, Garba, A, Stothard, JR, Gabrielli, AF, Clements, ACA, Kabatereine, NB, Toure, S, Dembele, R, Nyandindi, U, Mwansa, J and Koukounari, A. 2009. The Schistosomiasis Control Initiative (SCI): rationale, development and implementation from 2002-2008. Parasitology, 136: 17191730. DOI: https://doi.org/10.1017/S0031182009990400

Flanagin, AJ and Metzger, MJ. 2008. The credibility of volunteered geographic information. GeoJournal, 72: 137148. DOI: https://doi.org/10.1007/s10708-008-9188-y

Gomani, MC, Dietrich, O, Lischeid, G, Mahoo, H, Mahay, F, Mbilinyi, B and Sarmett, J. 2010. Establishment of a hydrological monitoring network in a tropical African catchment: An integrated participatory approach. Physics and Chemistry of the Earth, Parts A/B/C, 35: 648-656. DOI: https:// doi.org/10.1016/j.pce.2010.07.025

Gryseels, B, Polman, K, Clerinx, J and Kestens, L. 2006. Human schistosomiasis. The Lancet, 368(9541): 1106-1118. DOI: https://doi.org/10.1016/S0140-6736(13)61949-2

Hamer, SA, Curtis-Robles, R and Hamer, GL. 2018. Contributions of citizen scientists to arthropod vector data in the age of digital epidemiology. Current Opinion in Insect Science, 28: 98-104. DOI: https://doi.org/10.1016/j.cois.2018.05.005

Jacobs, L, Kabaseke, C, Bwambale, B, Katutu, R, Dewitte, O, Mertens, K, Maes, J and Kervyn, M. 2019. The geoobserver network: A proof of concept on participatory sensing of disasters in a remote setting. Science of The Total Environment, 670: 245-261. DOI: https://doi.org/10.1016/j. scitotenv.2019.03.177

Joan, N, Stephen, M, Bashir, M, Kiguli, J, Orikiriza, P, Bazira, J, Itabangi, $\mathbf{H}$ and Stanley, I. 2015. Prevalence and Economic Impact of Bovine Fasciolosis at Kampala City Abattoir, Central Uganda. British Microbiology Research Journal, 7: 109-117. DOI: https://doi.org/10.9734/BMRJ/2015/15274

Kabatereine, NB, Brooker, S, Koukounari, A, Kazibwe, F, Tukahebwa, EM, Fleming, FM, Zhang, Y, Webster, JP, Stothard, JR and Fenwick, A. 2007. Impact of a national helminth control programme on infection and morbidity in Ugandan schoolchildren. Bulletin of the World Health Organization, 11.

Kabatereine, NB, Brooker, S, Tukahebwa, EM, Kazibwe, F and Onapa, AW. 2004. Epidemiology and geography of Schistosoma mansoni in Uganda: implications for planning control. Tropical Medicine and International Health, 9: 372380. DOI: https://doi.org/10.1046/j.1365-3156.2003.01176.x

Kazibwe, F, Makanga, B, Rubaire-Akiiki, C, Ouma, J, Kariuki, C, Kabatereine, NB, Booth, M, Vennervald, BJ, Sturrock, RF and Stothard, JR. 2006. Ecology of Biomphalaria (Gastropoda: Planorbidae) in Lake Albert, Western Uganda: snail distributions, infection with schistosomes and temporal associations with environmental dynamics. Hydrobiologia, 568: 433-444. DOI: https://doi.org/10.1007/s10750-0060224-y

KoBoToolbox. 2014. KoBoToolbox | Data Collection Tools for Challenging Environments. Available at https://www. kobotoolbox.org/ (Last accessed 20 November 2020).

Krabbenhoft, CA and Kashian, DR. 2020. Citizen science data are a reliable complement to quantitative ecological assessments in urban rivers. Ecological Indicators, 116: 106476. DOI: https://doi.org/10.1016/j.ecolind.2020.106476

Maes, T, Hammoud, C, Volckaert, FAM and Huyse, T. 2021. A call for standardised snail ecological studies to support schistosomiasis risk assessment and snail control efforts. Hydrobiologia, 848(8): 1773-1793. DOI: https://doi. org/10.1007/s10750-021-04547-4

Malatji, MP, Pfukenyi, DM and Mukaratirwa, S. 2020. Fasciola species and their vertebrate and snail intermediate hosts in East and Southern Africa: a review. Journal of Helminthology, 94: e63. DOI: https://doi.org/10.1017/S0022149X19000531

Nieto, NC, Porter, WT, Wachara, JC, Lowrey, TJ, Martin, L, Motyka, PJ and Salkeld, DJ. 2018. Using citizen science to describe the prevalence and distribution of tick bite and exposure to tick-borne diseases in the United States. PLOS ONE, 13(7): e0199644. DOI: https://doi.org/10.1371/journal. pone.0199644

Perez-Saez, J, Mande, T, Zongo, D and Rinaldo, A. 2019. Comparative analysis of time-based and quadrat sampling in seasonal population dynamics of intermediate hosts of human schistosomes. PLOS Neglected Tropical Diseases, 13: e0007938. DOI: https://doi.org/10.1371/journal. pntd.0007938

Pocock, MJO, Roy, HE, August, T, Kuria, A, Barasa, F, Bett, J, Githiru, M, Kairo, J, Kimani, J, Kinuthia, W, Kissui, B, Madindou, I, Mbogo, K, Mirembe, J, Mugo, P, Muniale, FM, Njoroge, $\mathrm{P}$, Njuguna, EG, Olendo, MI, Opige, M, Otieno, TO, Ng'weno, CC, Pallangyo, E, Thenya, T, Wanjiru, A and Trevelyan, R. 2019. Developing the global potential of citizen science: Assessing opportunities that benefit people, society and the environment in East Africa. Journal of Applied Ecology, 56: 274-281. DOI: https://doi.org/10.1111/1365-2664.13279

Porter, WT, Motyka, PJ, Wachara, J, Barrand, ZA, Hmood, Z, McLaughlin, M, Pemberton, $K$ and Nieto, NC. 2019. Citizen science informs human-tick exposure in the Northeastern United States. International Journal of Health Geographics, 18: 9. DOI: https://doi.org/10.1186/s12942-019-0173-0 
Rowel, C, Fred, B, Betson, M, Sousa-Figueiredo, JC, Kabatereine, NB and Stothard, JR. 2015. Environmental Epidemiology of Intestinal Schistosomiasis in Uganda: Population Dynamics of Biomphalaria (Gastropoda: Planorbidae) in Lake Albert and Lake Victoria with Observations on Natural Infections with Digenetic Trematodes. BioMed Research International, 1-11. DOI: https://doi.org/10.1155/2015/717261

Shiff, C. 2017. Why reinvent the wheel? Lessons in schistosomiasis control from the past. PLOS Neglected Tropical Diseases, 11: e0005812. DOI: https://doi.org/10.1371/journal. pntd.0005812

Sokolow, SH, Huttinger, E, Jouanard, N, Hsieh, MH, Lafferty, KD, Kuris, AM, Riveau, G, Senghor, S, Thiam, C, N'Diaye, A, Faye, DS and De Leo, GA. 2015. Reduced transmission of human schistosomiasis after restoration of a native river prawn that preys on the snail intermediate host. Proceedings of the National Academy of Sciences, 112: 9650-9655. DOI: https:// doi.org/10.1073/pnas.1502651112

Sokolow, SH, Wood, CL, Jones, IJ, Lafferty, KD, Kuris, AM, Hsieh, MH and De Leo, GA. 2018. To Reduce the Global Burden of Human Schistosomiasis, Use 'Old Fashioned' Snail Control. Trends in Parasitology, 34: 23-40. DOI: https://doi. org/10.1016/j.pt.2017.10.002

Stothard, JR, Campbell, SJ, Osei-Atweneboana, MY, Durant, T, Stanton, MC, Biritwum, N-K, Rollinson, D, Ombede, DRE and Tchuem-Tchuenté, L-A. 2017. Towards interruption of schistosomiasis transmission in sub-Saharan Africa:
Developing an appropriate environmental surveillance framework to guide and to support 'end game' interventions. Infectious Diseases of Poverty, 6(1): 10. DOI: https://doi. org/10.1186/s40249-016-0215-9

Tchuem Tchuenté, L-A, Rollinson, D, Stothard, JR and Molyneux, D. 2017. Moving from control to elimination of schistosomiasis in sub-Saharan Africa: time to change and adapt strategies. Infectious Diseases of Poverty, 6: 42. DOI: https://doi.org/10.1186/s40249-017-0256-8

van der Wal, R, Sharma, N, Mellish, C, Robinson, A and Siddharthan, A. 2016. The role of automated feedback in training and retaining biological recorders for citizen science: Automated Feedback for Citizen Science. Conservation Biology, 30: 550-561. DOI: https://doi.org/10.1111/ cobi.12705

Weeser, B, Stenfert Kroese, J, Jacobs, SR, Njue, N, Kemboi, Z, Ran, A, Rufino, MC and Breuer, L. 2018. Citizen science pioneers in Kenya - A crowdsourced approach for hydrological monitoring. Science of The Total Environment, 631-632: 15901599. DOI: https://doi.org/10.1016/j.scitotenv.2018.03.130

West, S, Dyke, A and Pateman, R. 2021. Variations in the Motivations of Environmental Citizen Scientists. Citizen Science: Theory and Practice, 6: 14. DOI: https://doi. org/10.5334/cstp.370

World Health Organization. 2017. Field use of molluscicides in schistosomiasis control programmes: An operational manuel for programme managers. World Health Organization (WHO).

TO CITE THIS ARTICLE:

Brees, J, Huyse, T, Tumusiime, J, Kagoro-Rugunda, G, Namirembe, D, Mugabi, F, Nyakato, V, Anyolitho, MK, Tolo, CU and Jacobs, L. 2021. The Potential of Citizen-Driven Monitoring of Freshwater Snails in Schistosomiasis Research. Citizen Science: Theory and Practice, 6(1): 18, pp. 1-13. DOI: https://doi.org/10.5334/cstp.388

Submitted: 25 January 2021 Accepted: 11 June 2021 Published: 13 July 2021

\section{COPYRIGHT:}

(C) 2021 The Author(s). This is an open-access article distributed under the terms of the Creative Commons Attribution 4.0 International License (CC-BY 4.0), which permits unrestricted use, distribution, and reproduction in any medium, provided the original author and source are credited. See http://creativecommons.org/licenses/by/4.0/.

Citizen Science: Theory and Practice is a peer-reviewed open access journal published by Ubiquity Press.

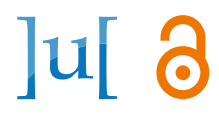

